\title{
Enhancement of Minority Carrier Lifetime of Fe Contaminated Boron-Phosphorus Compensated p-Type SoG Silicon
}

\author{
Mohammad Ziaur Rahman, ${ }^{* 1,2}$ and Mohammad Jahangir Alam ${ }^{1}$ \\ ${ }^{I}$ Department of Electrical and Electronic Engineering, Bangladesh University of Engineering and Technology, \\ Dhaka 1000, Bangladesh \\ ${ }^{2}$ Department of Electrical and Electronic Engineering, Ahsanullah University of Science and Technology, Dhaka \\ 141-142 Love Road, Tejgaon I/A, Dhaka 1208, Bangladesh
}

Received March 11, 2013; accepted April 10, 2013; published June 30, 2013

\begin{abstract}
To understand the role of deliberate phosphorus doping on the minority carrier lifetime of iron contaminated boron-phosphoruscompensated p-type solar grade silicon, a numerical study has been performed. This study confirmed that compensation results in a significant increase in the bulk lifetime of a minority carrier. The gain in the carrier lifetime is predicted due to the shift in the Fermi energy level, carriers screening and reduction in net equilibrium hole concentration. The bulk lifetime of a minority carrier reaches its maximum for a phosphorus concentration around $10^{15} \mathrm{~cm}^{-3}$ if the boron concentrations remain fixed at $10^{17} \mathrm{~cm}^{-3}$.
\end{abstract}

The aim of using lower grade silicon in the photovoltaic industries is to lower the module costs. Based on purity, three kinds of silicon are available, namely metallurgical grade $(98 \%$ pure), solar grade $(99.9999 \%$ pure) and electronic grade $(99.9999999 \%$ pure) silicon. The metallurgical silicon is not suitable for PV application. Although there might be slight differences in performance in comparison to $\mathrm{EG} \mathrm{Si}$, solar grade ( $\mathrm{SoG}$ ) silicon is the optimal choice to cut down the cost up to an acceptable minimum as it is the cheapest among the three types of the above mentioned graded silicon. The main issue involved with the SoG is the amount of impurities (e.g. Fe, $\mathrm{Cr}, \mathrm{Ni}$, etc.) present. Impurities are found to be detrimental to the final solar cell conversion efficiency. The transition metal impurities are likely to affect the minority carrier lifetime, thus affecting the efficiency of the solar cells made out of SoG silicon. Due to their higher segregation coefficient, the complete removal of electrically active impurities of boron (B) and phosphorus (P), using conventional purification techniques, such as the Siemens process, are quite challenging, meaning that SoG is often compensated. Both $\mathrm{B}$ and $\mathrm{P}$ introduce shallow energy levels (B at Ev+0.045 eV and P at Ec-0.044 eV) [1] near the energy bands and act as either recombination or trap centres. These shallow energy levels enhance the recombination lifetime of minority carrier.

\footnotetext{
*E-mail: ziaeee_083@yahoo.com
}

Recent studies explain the transport properties and impact of boron-oxygen related defects in compensated silicon [2-4]. Dubois et al. [5] studied the effect of compensation level on a carrier lifetime. Recently Macdonald and Cuevas [6] studied the recombination in compensated silicon for solar cells.

Previous studies showed that compensation would lead to an increase in the carrier scattering mechanism, lower the carrier mobilities, and hence could limit the lifetime in highly doped SoG Si specially after a phosphorus diffusion step [7]. This study finds that it is possible to increase both intrinsic and the Shockley-Read-Hall (SRH) recombination lifetime of a minority carrier significantly by virtue of suitable boron-phosphorus compensation.

There is a direct correlation between the carrier lifetime and conversion efficiency of a solar cell [8]. Extensive research endeavor was made over the last few decades to ameliorate the factors affecting the lifetime to upgrade the efficiency of both single doped mono and multi-crystalline silicon solar cells. We have deployed those previously published relevant data to study the impact of compensation on minority carrier lifetime. By 'lifetime' we have meant recombination lifetime due to the results of radiative, Auger and SRH recombination processes [9].

This study uses the analytical equations available for different recombination mechanisms at low injection and low temperature [8-9]. The equations are slightly modified to incorporate the compensation effect. The presence of interstitial iron introduces a defect centre at the energy level $\mathrm{Ev}+0.38 \mathrm{eV}$. Iron-boron pair introduces an energy level at Ec- $0.23 \mathrm{eV}$ and Ec-0.29eV [10]. These energy levels are mainly responsible for SRH recombination [11-12]. Compensation reduces the net doping concentration, and has great influences on intrinsic recombination processes [6].

For a fixed acceptor doping of $N_{A}=10^{17} \mathrm{~cm}^{-3}$, donor concentration, $N_{D}$ is varied over a range of $10^{14}$ to $10^{16}$ so that it becomes predominantly p-type. Net hole 
concentration, $\mathrm{p}_{0}$, for $\mathrm{p}$-type compensated silicon is the difference between $N_{A}$ and $N_{D}$.

At low injection, radiative recombination is inversely proportional to net doping while Auger recombination is inversely proportional to the square of the net doping [89]. In uncompensated p-type silicon, the net doping is simply equal to $N_{A}$. In uncompensated silicon the impact of intrinsic recombination in carrier lifetime is negligible (shown in Fig. 1), but our simulated results showed it is not the same case for compensated silicon. There is a substantial increase (about 100 times) in minority carrier lifetime for low level compensation and is shown in Fig.2. Due to the compensation of boron by phosphorus atoms, the reduction in a free carrier shifts the Fermi level near the mid gap of an energy band. As a result, the attractivity of neutral boron becomes exacerbated for the electrons. Coulomb interaction between quasi-free particles results in local inhomogeneities in the carrier density and induces a spatial correlation of electron-hole pairs due to the formation of bound states. These bound states dominate under low doping concentration and are found to be vanished at a doping concentration above $10^{18} \mathrm{~cm}^{-3}$ because of complete screening of electron-hole interaction [13]. The overall impact is an increase in the minority carrier lifetime.

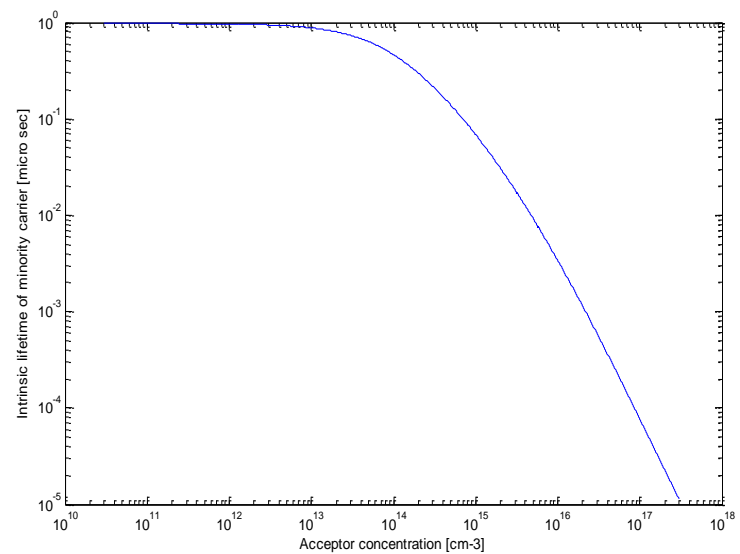

Fig. 1. Intrinsic carrier lifetime of minority carrier in uncompensated SoG Silicon.

At low injection, standard SRH lifetime equation [8-9] is modified into

$$
\tau_{S R H}=\left(N_{t} \sigma_{n} v_{t h}\right)^{-1}\left[\left(\frac{p_{0}+p_{1}}{p_{0}+n_{0}}\right)+\frac{\sigma_{n}}{\sigma_{p}}\left(\frac{n_{0}+n_{1}}{p_{0}+n_{0}}\right)\right],
$$

where $N_{t}$ is defect concentration, $v_{t h}$ is thermal velocity, $\sigma_{\mathrm{n}}$ $\left(1-1.4 \cdot 10^{-14} \mathrm{~cm}^{2}\right)$ and $\sigma_{\mathrm{p}}\left(6-7 \cdot 10^{-17} \mathrm{~cm}^{2}\right)$ are electron and hole capture cross section, respectively, $p_{0}$ and $n_{0}$ are equilibrium hole and electron density respectively, $p_{1}$ and $n_{1}$ are hole and electron concentration calculated at defect energy level.

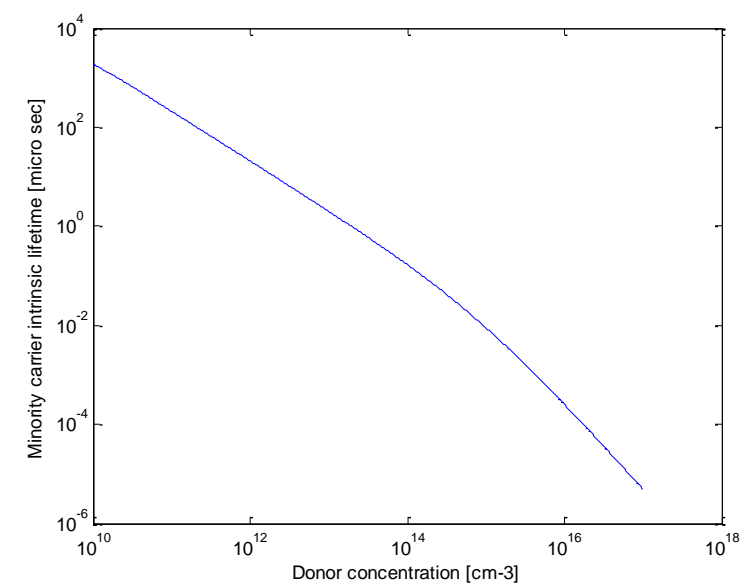

Fig. 2. Intrinsic carrier lifetime of a minority carrier in compensated SoG Silicon.

For p-type compensated silicon, Eq. (1) can be further reduced as follows

$$
\tau_{S R H}=\left(p_{0} N_{t} \sigma_{n} v_{t h}\right)^{-1}\left[\left(p_{0}+p_{1}\right)+\frac{\sigma_{n}}{\sigma_{p}} n_{1} p_{0}\right] .
$$

To avoid the trapping effect, $N_{\mathrm{t}}$ is set equal to $10^{12} \mathrm{~cm}^{-3}$. The defect energy levels introduced by iron play a vital role in $\mathrm{SRH}$ recombination. Interstitial iron $\left[\mathrm{Fe}_{\mathrm{i}}\right]$ that introduces energy level close to a mid-gap is most effective for SRH recombination. For a fixed value of the hole concentration, if the donor concentration varies then the hole is screened out by electron. For this reason, equilibrium hole concentration, $p_{0}$ decreases. As a result, $\mathrm{SRH}$ lifetime increases as the compensation level increases as illustrated in Fig. 3.

The compensation ratio is defined as

$$
R_{c}=\frac{N_{A}+N_{D}}{N_{A}-N_{D}}
$$

where $N_{A}$ and $N_{D}$ are the acceptor and donor concentration, respectively. The analytical model adopted in the presented study has found an increase of $0.2 \mathrm{~ms}$ in SRH lifetime for a factor of 10 change in compensation ratio as shown in Fig. 4. 


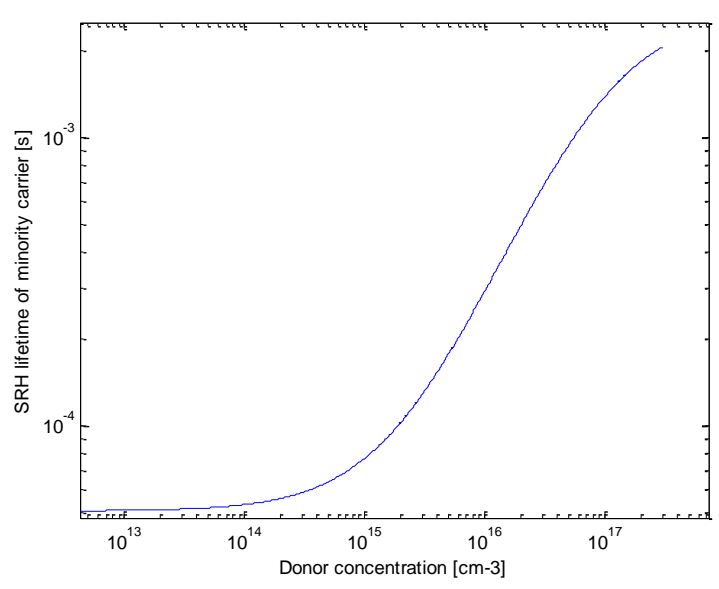

Fig. 3. Minority carrier lifetime for SRH recombination of compensated SoG silicon.

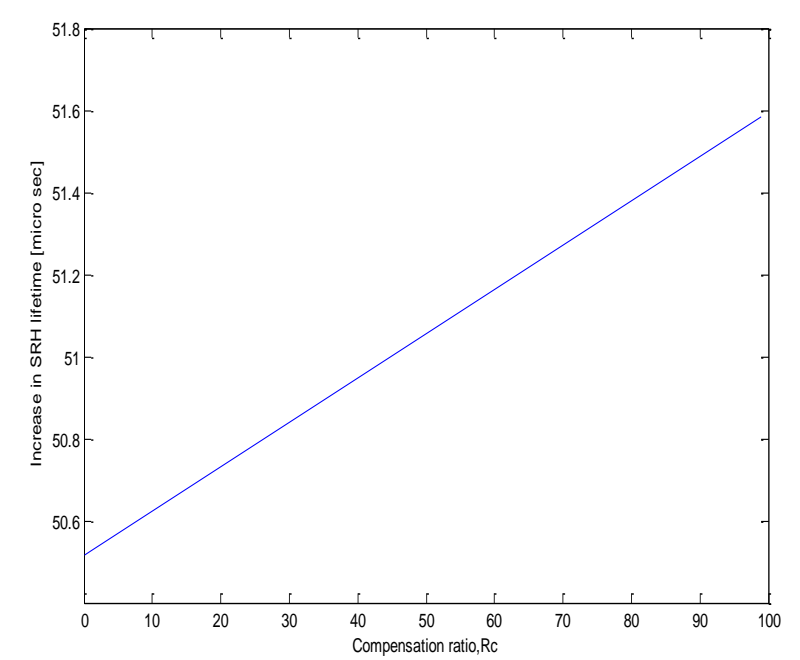

Fig. 4. Gain in SRH lifetime.

The focus of the research was only to deal with bulk recombination mechanisms associated with SoG silicon. The overall impact of compensation on bulk lifetime can be modeled as

$$
\tau_{b u l k}=\frac{\tau_{S R H} \tau_{i}}{\tau_{S R H}+\tau_{i}},
$$

where $\tau_{i}$ is the intrinsic carrier lifetime. For deep energy level, SRH recombination lifetime becomes almost equal to a minority carrier capture time constant and then the dopant concentration has less significance on $\tau_{S R H}$. In this case, $\tau_{\text {bulk }} \cong \tau_{S R H}$. It can also be confirmed from the simulated results shown in Fig. 5.

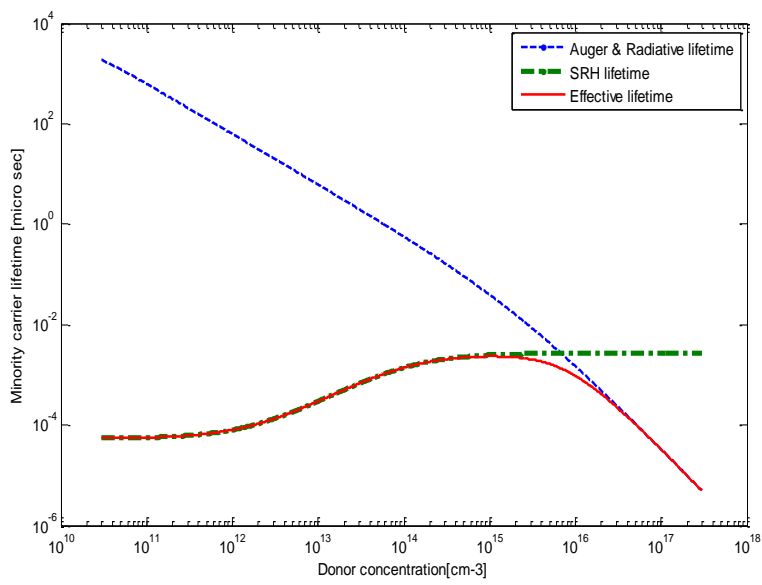

Fig. 5. Bulk lifetime of minority carrier in compensated SoG silicon.

From Fig. 5, it is clear that the bulk recombination lifetime is dominated by SRH recombination lifetime for a lower compensation, while it follows the same pattern of intrinsic lifetime for donor concentrations above $10^{16} \mathrm{~cm}^{-3}$. The maximum of bulk lifetime occurs for a donor concentration around $10^{15} \mathrm{~cm}^{-3}$ for a fixed acceptor concentration of $10^{17} \mathrm{~cm}^{-3}$.

This study confirmed that a suitable donor compensation may results in an upgrade in both intrinsic and SRH lifetime. The increase in a minority carrier bulk lifetime is quite significant and noticeable.

We would like to extend our heartiest thanks to our anonymous reviewers whose comments helps us a lot to improve our manuscript in the present form. We are also grateful to the editor for her kind cooperation.

\section{References}

[1] M.A. Green, Solar cells- operating principles, technology and system applications (Prentice-Hall 1986).

[2] F.E. Rougieux, D. Macdonald, A. Cuevas, Prog. Photovolt: Res. Appl. 19(7), 787 (2011).

[3] F.E. Rougieux, M. Forster, D. Macdonald, A. Cuevas, B. Lim, J. Schmidt, IEEE Journal of Photovoltaics 1, 54 (2011).

[4] D. Leblanc, K. Putyera, Trans.Nonferrous Met. Soc. China 21, 1172 (2011).

[5] S. Dubois, N. Enjalbert, J.P. Garandet, Appl. Phys. Lett. 93, 032114 (2008).

[6] D. Macdonald, A. Cuevas, J. Appl. Phys. 109, 043704 (2011).

[7] J. Veirman, S. Dubois, N. Enjalbert, J.P. Garandet, M. Lemiti, J. Appl. Phys. 109, 103711 (2011).

[8] D. Macdonald, PhD Thesis (Australian National University 2001).

[9] S. Rein, Lifetime Spectroscopy (Springer 2005).

[10] A. A. Istratov, H. Hieslmair, E. R. Weber, Appl. Phys. A: Mater. Sci. Process. 69, 13 (1999).

[11] W. Shockley, W. T. J Read, Phys. Rev. 87 (5), 835 (1952).

[12] R.N. Hall, Phys. Rev. 87, 387 (1952).

[13] A. Hangleiter, R. Hacker, Phys. Rev. Lett. 65 (2), 215 (1990). 